\title{
Characterization of Intermetallic Precipitates Observed in 7XXX Series Aluminum Alloys Containing Manganese Using Aberration Corrected STEM
}

\author{
Robert E A Williams
}

THE Ohio State University, Columbus, Ohio, United States

Intermetallic precipitates found in 7xxx series $\mathrm{Al}$ alloys, such as $\mathrm{Al}-\mathrm{Zn}-\mathrm{Mg}-\mathrm{Mn}$ alloys, can have a marked effects on mechanical properties and depending on thermomechanical history, may have a positive or deleterious effect. Two intermetallic precipitate phases, Al57Mn12 and Al6Mn, are believed to play an important role in enhancing 7XXX series Al alloy's strength. Microstructural parameters such as precipitate volume fraction, size, shape, and spatial distribution determine the relative effectiveness of precipitation hardening achieved, however characterization of these precipitates has proven challenging[1]. Identification of various Mn-containing precipitates has been performed by classical electron diffraction to determine orientation relationships, but this characterization technique does not allow for individual precipitate phase identification. Typically, intermetallic precipitates need to remain small to provide positive strengthening benefits and this makes uniquely identifying the small intermetallic precipitates through experimental characterization of three-dimensional precipitate morphology and shape difficult. To overcome this, concurrent imaging and phase identification of the various precipitate are required to correlate the observed two-dimensional cross section to the correct intermetallic precipitate.

Inherent in TEM imaging are thin foils, and often intermetallic precipitates will have a dimension that extend past the TEM foil dimensions resulting in varied, observed two-dimensional cross sections; this discrepancy has resulted in conflicting results and researchers have speculated as to the actual threedimensional shape to explain varied two-dimensional cross sections observed by TEM, with little consensus reached as multiple intermetallic precipitates are observed in a field of view. Currently, differentiating and uniquely identifying the small intermetallic precipitates is compounded due to difficulty in collecting quantifiable spectroscopy signals to confirm the small, intermetallic precipitate compositions. In order to predict three-dimensional precipitate morphology, phase field modelling will be used in conjunction with current understanding of precipitate growth models to produce various size precipitates that can be two dimensionally cross sectioned to reveal correlate predicted precipitate morphologies to those observed experimentally. Three-dimensional morphologies of the Mn-containing precipitates observed in 7XXX series Al alloys is unclear at this time, this work will discuss methods for precipitate identification using HAADF-STEM imaging, image simulation and XEDS characterization, coupled with phase field modelling to help explain why the observed intermetallic precipitates have drastically different shapes and provide a modern characterization method for uniquely identifying and characterizing intermetallic precipitates in 7XXX series Al Alloys containing Mn. This work was performed at the Center for Electron Microscopy(CEMAS) at THE Ohio State University.

\section{References}

[1] Brown LM, Ham RK. In: Kelly A, Nicholson RB, editors. Strengthening methods in crystals. London: Elsevier; 1971. 